\title{
Molecular dynamics of the $\alpha$ relaxation during crystallization of a glassy polymer: A real-time dielectric spectroscopy study
}

\author{
T.A. Ezquerra, J. Majszczyk, ${ }^{*}$ and F.J. Baltà-Calleja \\ Instituto de Estructura de la Materia, Consejo Superior de Investigaciones Científicas, Serrano 119, Madrid 28006, Spain \\ E. López-Cabarcos \\ Facultad de Farmacia, Universidad Complutense de Madrid, Madrid, Spain \\ K.H. Gardner and B.S. Hsiao \\ Central Research and Development, E.I. DuPont de Nemours, Experimental Station, Wilmington, Delaware $19880-0356$
}

(Received 3 March 1994)

\begin{abstract}
The change of the $\alpha$ relaxation process occurring during isothermal crystallization of a glassy polymer, poly(aryl ether ketone ketone), has been followed in real time through measurement of the dielectric complex permittivity. The dielectric strength has been correlated with the degree of crystallinity derived from real time wide angle $x$-ray patterns using synchrotron radiation. The phenomenological Havriliak-Negami description has been used to analyze the changes of the dielectric strength, central relaxation time and shape parameters describing the relaxation, as crystallization proceeds. The evolution of the dielectric magnitudes with crystallization time has been interpreted in the light of the Schönhals-Schlosser model. According to this model, a restriction of the long scale motions of the polymeric chains as the material is filled in with crystals is observed. The derived dipole moment time correlation functions have been calculated for different crystallization times and fitted by the Kohlrausch-Williams-Watts function. The variation of the stretching parameters with crystallization time can be interpreted in terms of the coupling model assuming an increase of the intermolecular cooperativity of the $\alpha$ relaxation as the polymeric chains are constrained to move between crystalline regions. These results offer a contribution to the understanding of molecular dynamics of a glassy polymer during crystallization.
\end{abstract}

\section{INTRODUCTION}

Glass-forming liquids and polymers are considered to be complex systems. The existence of complexity is reflected by the fact that the relaxation of a measured magnitude deviates from a single linear exponential Debye behavior characterized by a single relaxation time. The phenomenological Havriliak-Negami (HN) formulation ${ }^{1}$ has been widely used to describe, in the frequency domain, the non-Debye character of the $\alpha$ relaxation which appears in glass-forming liquids and polymeric systems at temperatures above the glass transition temperature and involve motions extended to several molecular segments. By using the Havriliak-Negami (HN) relaxation function, both dielectric and mechanical relaxation as well as nuclear magnetic resonance and neutron scattering experiments can be satisfactorily interpreted. ${ }^{2,3}$ As far as dielectric relaxation is concerned, the HavriliakNegami formulation assumes a frequency $(\omega)$ dependence of the complex dielectric permittivity of the form ${ }^{1}$

$$
\epsilon^{*}=\left(\Delta \epsilon^{*}\right)+\left(\epsilon_{\infty}\right),
$$

with

$$
\Delta \epsilon^{*}=\frac{\epsilon_{0}-\epsilon_{\infty}}{\left[1+\left(i \omega \tau_{0}\right)^{b}\right]^{c}},
$$

where $\epsilon_{0}$ and $\epsilon_{\infty}$ are the relaxed $(\omega=0)$ and unrelaxed $(\omega=\infty)$ dielectric constant values, $\tau_{0}$ is the central relaxation time of the relaxation time distribution function, and $b$ and $c$ are shape parameters which describe the symmetric and the asymmetric broadening of the relaxation time distribution function, respectively. ${ }^{1}$ According to a recent model proposed by Schönhals and Schlosser (SS), the shape parameters can be related to the molecular dynamics at the glass transition provided that the molecular mobility is controlled by inter- and intramolecular interactions. ${ }^{4}$ The model relates the $b$ parameter to the large scale motions and the $b c$ product to the small scale motions. Experimental support of the model has been provided by different dielectric spectroscopy techniques. ${ }^{5}$

In the time domain, the non-Debye behavior of the $\alpha$ relaxation is characterized by the time dependence of the macroscopic decay function, which is described by the Kohlrausch-Williams-Watts function ${ }^{6}$ (KWW)

$$
\phi(t) \propto \exp \left[-\left(t / \tau_{\mathrm{KWw}}\right)^{\beta}\right]
$$

where $\tau_{\mathrm{KWw}}$ is a characteristic relaxation time, and $\beta$ takes values between 0 and 1 . The model ${ }^{7,8}$ proposed by Ngai et al. to explain the physical basis of the KWW stretched exponential considers the coupling of relaxing species with neighboring nonbonded ones as the 
main reason for complexity. This intermolecular coupling or degree of cooperative complexity provokes a slowing down of the segmental relaxation giving rise to a $\mathrm{KWW}$ decay function. The degree of coupling is characterized by a new parameter $n$ related to the experimentally observed $\beta$ value by $\beta=1-n$. The KWW stretched exponential describes with a single parameter, $\beta$, experiments in the time domain. The relationships between the $b$ and $c$ parameters of the $\mathrm{HN}$ formulation, describing a relaxation process in the frequency domain, and the corresponding $\beta$ parameter in Eq. (3), which describes the same process in the time domain, have been the subject of attention in several laboratories. ${ }^{9-11}$

It is known that some glass-forming polymeric systems develop a certain level of crystallinity provided they are heated at temperatures above the glass transition temperature $\left(T_{g}\right)$. The kinetics of crystallization depends on the crystallization temperature. ${ }^{12}$ In a typical isothermal crystallization process of an initially glassy polymeric system, crystallinity evolves in a sigmoidal fashion. ${ }^{13}$ After an initial time interval where no crystallinity development is observed, crystallization takes place at a given rate until a certain regime is achieved where the crystallization rate is strongly reduced. A half crystallization time can be taken as a measure characterizing the overall rate of crystallization.

In semicrystalline polymers at temperatures higher than the glass transition temperature, some of the long amorphous polymeric chains are restricted to move between the crystalline regions. ${ }^{14}$ This chain confinement modifies the dynamics of the $\alpha$ relaxation process and is characterized by a decrease of the dielectric strength $\left(\Delta \epsilon=\epsilon_{0}-\epsilon_{\infty}\right)$, an increase of the central relaxation time $\left(\tau_{0}\right)$, and a concurrent change of the shape parameters. ${ }^{15-17}$

Until now the majority of studies related to crystallization and dielectric relaxation have been carried out on isothermally crystallized materials from the glassy state after subsequent quenching. Only a few measurements have been performed during the real-time of crystallization. This is so because the time of crystallization often interferes with the time required for measurement. Recently, the use of impedance analyzers ${ }^{18}$ capable of measuring over several decades of frequency during measuring times less than 1 min allows one to perform dielectric measurements during crystallization in real time.

With the above ideas in mind in the present paper we have studied the dielectric behavior of an initially glassy polymer [poly(aryl ether ketone ketone) (PEKK)] by measurements performed in real time as crystallization proceeds. Our aim is to establish a relationship between the change of the shape parameters and the restriction on the overall chain mobility imposed by crystallization. The study of the real-time variation, in the frequency domain, of the shape parameters describing the dielectric relaxation of the $\alpha$ process upon crystallization allows a direct comparison with the predictions of the SSmodel. Concurrently, from the evolution of the derived decay functions, in the time domain, as crystallization proceeds, an interpretation based on the coupling model is attempted.

\section{THEORETICAL PART}

According to the HN approach, Eq. (1) can be used to derive the corresponding analytical expressions of the real and imaginary parts of the dielectric permittivity ${ }^{1}$ which read

$$
\begin{aligned}
& \epsilon^{\prime}=\left(\epsilon_{0}-\epsilon_{\infty}\right) r^{-c} \cos (c \psi)+\epsilon_{\infty}, \\
& \epsilon^{\prime \prime}=\left(\epsilon_{0}-\epsilon_{\infty}\right) r^{-c} \sin (c \psi),
\end{aligned}
$$

with

$$
r^{2}=1+2\left(\omega \tau_{0}\right)^{b} \cos (b \pi / 2)+\left(\omega \tau_{0}\right)^{2 b}
$$

and

$$
\tan \psi=\frac{\left(\omega \tau_{0}\right)^{b} \sin (b \pi / 2)}{1+\left(\omega \tau_{0}\right)^{b} \cos (b \pi / 2)} .
$$

The dielectric complex permittivity $\epsilon^{*}$ is related to the macroscopic decay function $\phi(t)$ by a pure imaginary Laplace transformation ${ }^{6,14,19}$ of the form

$\frac{\epsilon^{*}-\epsilon_{\infty}}{\epsilon_{0}-\epsilon_{\infty}}=\mathcal{L}\left(-\frac{d \phi(t)}{d t}\right)=\int_{0}^{\infty} \exp (-i \omega t)\left[-\frac{d \phi(t)}{d t}\right] d t$

Through the knowledge of $\phi(t)$ over the entire time range the values of $\left(\epsilon^{*}-\epsilon_{\infty}\right) /\left(\epsilon_{0}-\epsilon_{\infty}\right)$ can be derived. Inversely, it can be shown that

$$
\phi(t)=\frac{2}{\pi} \int_{0}^{\infty}\left(\frac{\epsilon^{\prime \prime}(\omega)}{\epsilon_{0}-\epsilon_{\infty}}\right) \cos (\omega t) \frac{d \omega}{\omega} .
$$

For materials of low permittivity, $\epsilon_{0} \approx \epsilon_{\infty}$, the macroscopic decay function can be considered to be approximately the same as the dipole moment time correlation function ${ }^{14,20}$

$$
\bigwedge(t)=\frac{\sum_{i}^{N} \sum_{j}^{N}\left\langle\mu_{i}(0) \mu_{j}(t)\right\rangle}{\sum_{i}^{N} \sum_{j}^{N}\left\langle\mu_{i}(0) \mu_{j}(0)\right\rangle}
$$

where $\mu_{j}(t)$ is the elemental dipole moment at a given time. Experimentally, the shape of $\phi(t)$ has been described in terms of the KWW stretched exponential function [Eq. (3)]. The mean relaxation time within this representation is given by

$$
\langle\tau\rangle=\left[\frac{\tau_{\mathrm{KWW}}}{\beta} \Gamma\left(\frac{1}{\beta}\right)\right],
$$

where $\Gamma$ is the gamma function.

\section{EXPERIMENTAL PART}

Poly(aryl ether ketone ketone) (PEKK) is a rigid polymer from the poly (aryl ether ketone) family whose generalized chemical structure is shown in Fig. 1. PEKK can be prepared from diphenyl ether, terephthalic acid ( $T$ ), and isophthalic acid (I). By controlling the amount of $\mathrm{T}$ and I components, polymers with different para/meta 


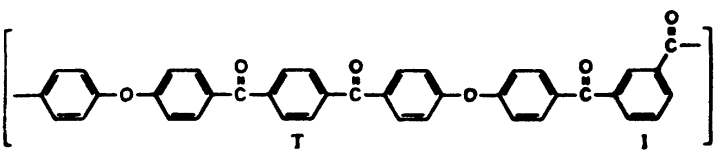

FIG. 1. Chemical structure of a poly(aryl ether ketone ketone) polymer (PEKK).

phenyl isomer ratios can be prepared. The $\mathrm{T} / \mathrm{I}$ ratio modifies notably the melting temperature as well as the crystallization kinetics. However the $\mathrm{T} / \mathrm{I}$ ratio has only a small effect on the glass transition temperature. ${ }^{21}$ In the present work a 60/40 PEKK specimen, synthesized at DuPont, with a $T_{g}=428 \mathrm{~K}$ has been investigated. The crystallization kinetics of this polymer allows one to keep the amorphous state at temperatures $T>T_{g}$ where the maximum loss of the $\alpha$ relaxation process appears within our experimental frequency window. The original samples were amorphous films with a thickness of about 0.01 cm. Measurements of the dielectric complex permittivity $\left(\epsilon^{*}=\epsilon^{\prime}-i \epsilon^{\prime \prime}\right)$ were performed in the $10^{3}-10^{6} \mathrm{~Hz}$ frequency range using a Hewlett-Packard impedance analyzer HP 4192A. Films with circular gold electrodes (4 $\mathrm{cm}$ diameter) were prepared by sputtering the metal in both free surfaces.

The specimens were placed between two gold-plated stainless steel electrodes. The dielectric cell was introduced in a homemade cryostat operating in a temperature controlled nitrogen atmosphere. To perform isothermal crystallization measurements the sample was brought to the crystallization temperature at a constant heating rate of $1^{\circ} \mathrm{C} / \mathrm{min}$. After reaching the selected $T_{c}$, $\epsilon^{*}$ measurements were performed in the $10^{3}-10^{6} \mathrm{~Hz}$ frequency range as a function of crystallization time. Each measurement required about $40 \mathrm{~s}$. The experimental conditions were carefully chosen to avoid significant precrystallization of the sample during the heating process. ${ }^{21}$

Wide angle $x$-ray scattering experiments were performed using a double-focusing mirror monochromator on the polymer beam line at HASYLAB (Hamburg, F.R.G.). The samples were heated in vacuum (10 $10^{-2}$ torr) at the same heating rate as in the dielectric experiments. Thermal contact and homogeneous heating were ensured by a thin aluminum foil covering the surfaces of the film. The temperature was measured by a thermocouple inserted in the sample. The wavelength used was $0.15 \mathrm{~nm}$ with a bandpass of $\Delta \lambda / \lambda=5 \times 10^{-3}$. After reaching the selected crystallization temperature $T_{c}$, scattering patterns were recorded every $60 \mathrm{~s}$ using a linear positionsensitive detector and corrected for fluctuations in the intensity of the primary beam and background. The data acquisition system is based on CAMAC hardware and modulator software ${ }^{22}$

\section{RESULTS}

\section{A. Dielectric relaxation of the glassy polymer}

Dielectric loss $\left(\epsilon^{\prime \prime}\right)$ and dielectric constant $\left(\epsilon^{\prime}\right)$ values at $10^{3} \mathrm{~Hz}$ measured for the initially amorphous $60 / 40$ sample as a function of the temperature are illustrated in Figs. 2(a) and 2(b), respectively. Two main dielectric relaxations, labeled as $\alpha$ and $\beta$ in the order of decreasing temperatures, can be distinguished in the investigated temperature range. The frequency of the maximum loss $\left(\nu_{\max }\right)$ is found to increase for both processes as the temperature increases. Values of $\log _{10} \nu_{\max }$ as a function of the reciprocal temperature are shown as an inset in Fig. 2(a). According to Sauer et al. ${ }^{23}$ the $\beta$ process can be associated with the local motions of the ketone group and the $\alpha$ process is related to the larger scale motions appearing at temperatures above $T_{g}$. The $\beta$ process follows an Arrhenius behavior. The activation energy, derived from the slope, gives the value af $13.8 \pm 2.5 \mathrm{kcal} / \mathrm{mol}$. This value is in good agreement with those previously reported. ${ }^{23}$ The solid line shown in the inset of Fig. 2(a) for the $\alpha$ process has been obtained by fitting the experimental results for the $60 / 40$ polymer to the Vogel-Fulcher equation

$$
\log _{10}\left\langle\nu_{\max }\right\rangle=B+\frac{A}{T-T_{0}},
$$
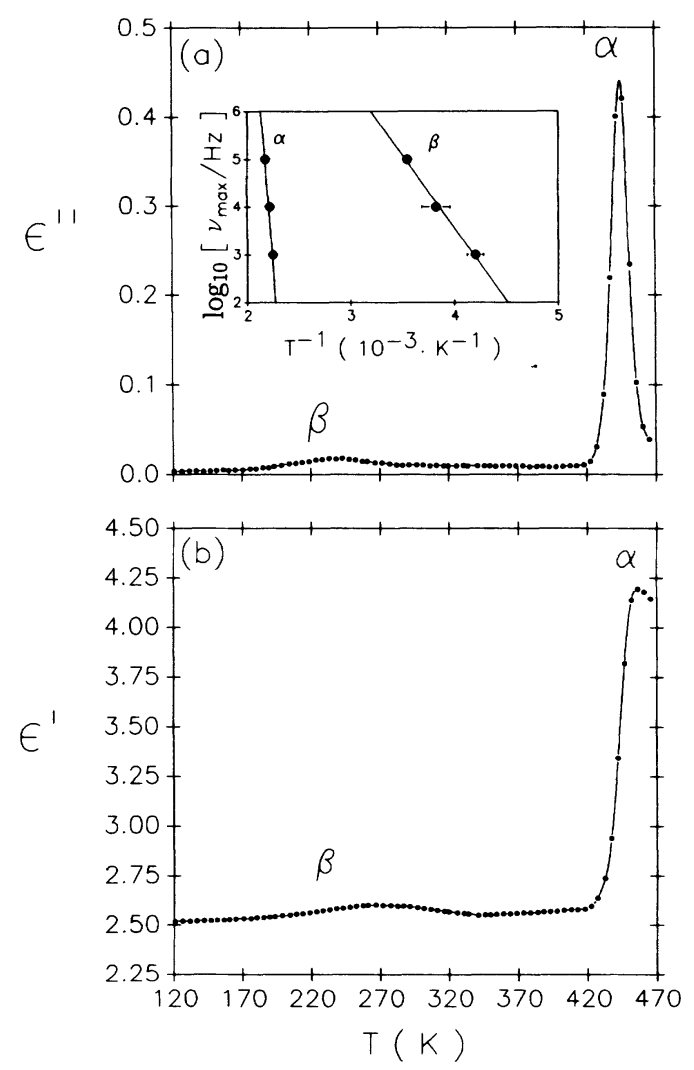

FIG. 2. (a) Dielectric loss, $\epsilon^{\prime \prime}$, and (b) dielectric constant, $\epsilon^{\prime}$, for the amorphous PEKK sample as a function of temperature at $10^{3} \mathrm{~Hz}$. The inset shows the logarithm of the frequency of maximum loss, $\nu_{\max }$, as a function of the reciprocal temperature for the $\alpha$ and $\beta$ relaxation. 
where $A, B$, and $T_{0}$ are constants characteristic for the system. The fitting parameters obtained are $A=$ $-1301.2, B=17.47$, and $T_{0}=356.5 \mathrm{~K}$.

\section{B. Real-time variation of the $\alpha$ relaxation process upon crystallization}

Figure 3 shows the real-time evolution of the $\alpha$ relaxation during an isothermal crystallization process at $T_{c}=461 \pm 1.5 \mathrm{~K}$ for the investigated polymer. Figures $3(\mathrm{a})$ and $3(\mathrm{~b})$ show the $\epsilon^{\prime \prime}$ and $\epsilon^{\prime}$ data respectively. In Fig. 3(a) every single curve presents the dependence of $\epsilon^{\prime \prime}$ versus frequency at a selected crystallization time which is labeled on the right side of the figure. The initial curve, at $t=0$, corresponds to the initially amorphous polymer. As crystallization proceeds two main features are observed in Fig. 3(a). First, there is a reduction of the intensity of the $\alpha$ relaxation process as the crystallization time increases. Second, a shift of the frequency of maximum loss towards lower frequency values is detected. Figure 4 shows the plot of the logarithm of the maximum

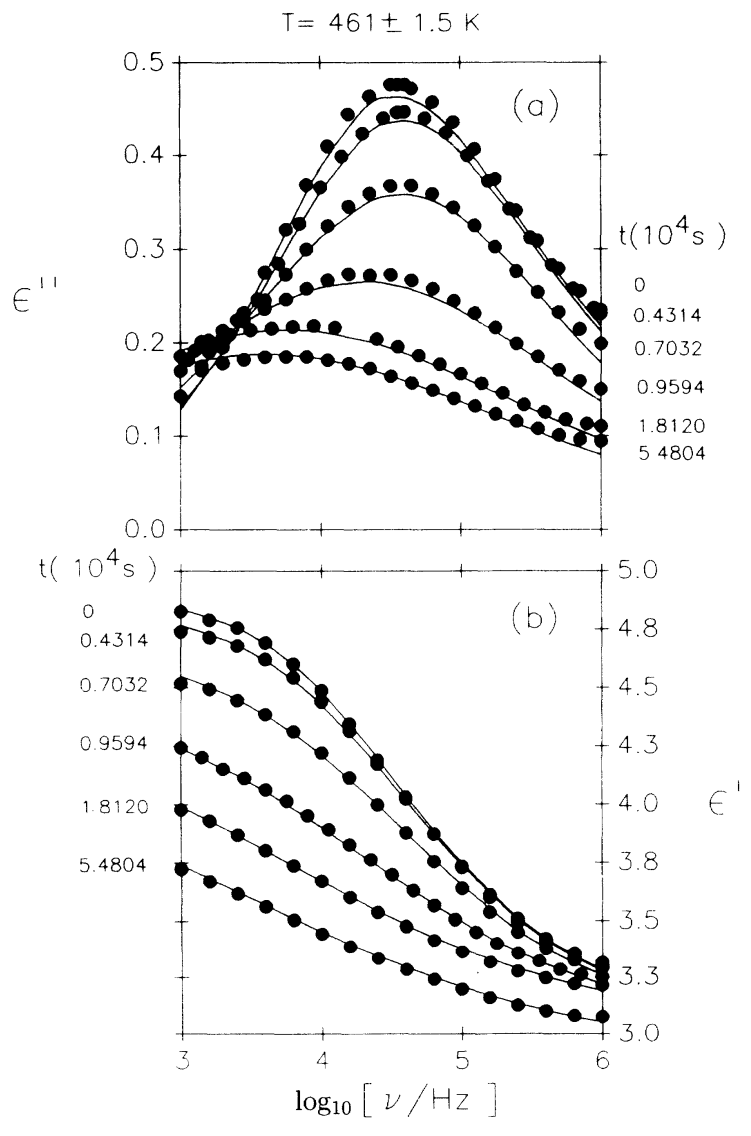

FIG. 3. Real-time evolution of the $\alpha$ relaxation dielectric loss, $\epsilon^{\prime \prime}(\mathrm{a})$, and dielectric constant, $\epsilon^{\prime}(\mathrm{b})$, values versus frequency during an isothermal crystallization process at $T=461 \pm 1.5 \mathrm{~K}$ at selected crystallization times. The continuous lines are $\mathrm{HN}$ fits according to Eqs. (4)-(7). Fitting parameters are shown in Fig. 9.

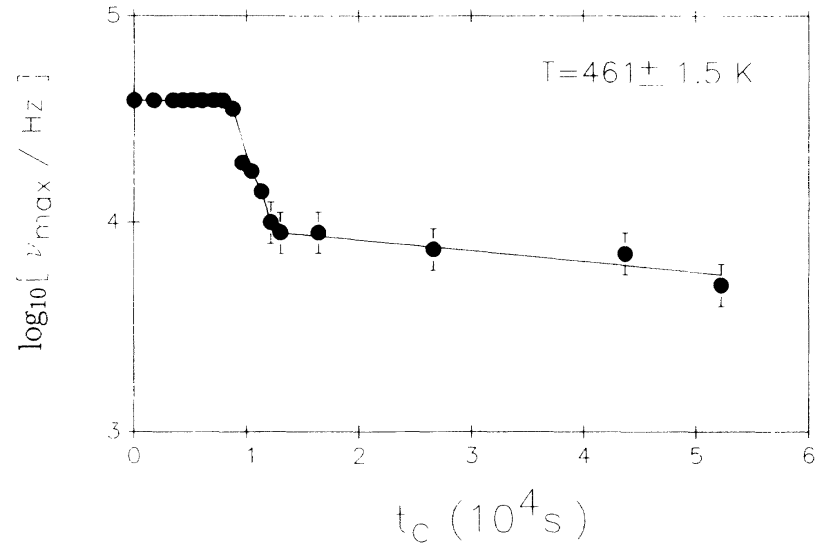

FIG. 4. Variation of the frequency of maximum loss values, $\nu_{\max }$, corresponding to Fig. 3(a) as a function of crystallization time $\left(t_{c}\right)$.

loss frequency, $\nu_{\max }$, as a function of the crystallization time. If one considers that $\left(2 \pi \nu_{\max }\right)^{-1}$ is an average relaxation time ${ }^{14}$ the observed decrease of $\nu_{\max }$ suggests a slowing down of the overall chain mobility as crystallization occurs.

\section{Real-time x-ray diffraction study during crystallization}

Figure 5 shows the variation of the wide angle $\mathrm{x}$ ray scattering (WAXS) pattern in real time during isothermal crystallization of the investigated polymer

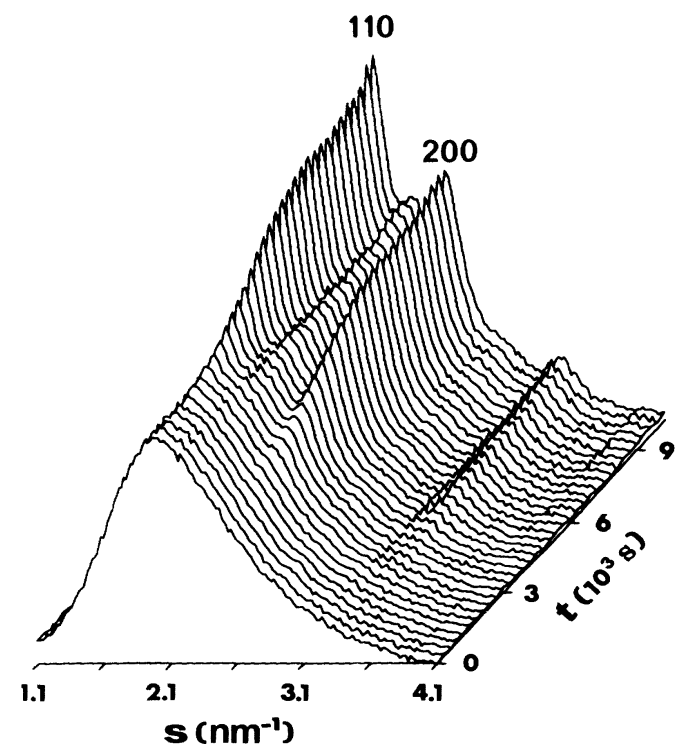

FIG. 5. Three-dimensional plot showing the real-time evolution of the WAXS patterns with crystallization time $\left(t_{c}\right)$ at $T_{c}=461 \pm 1.5 \mathrm{~K}$ of the PEKK $(60 / 40)$ system. The diffracted $\mathrm{x}$-ray intensity has been represented as a function of the reciprocal lattice vector $s=(2 \sin \theta) / \lambda$ for each time in seconds. 


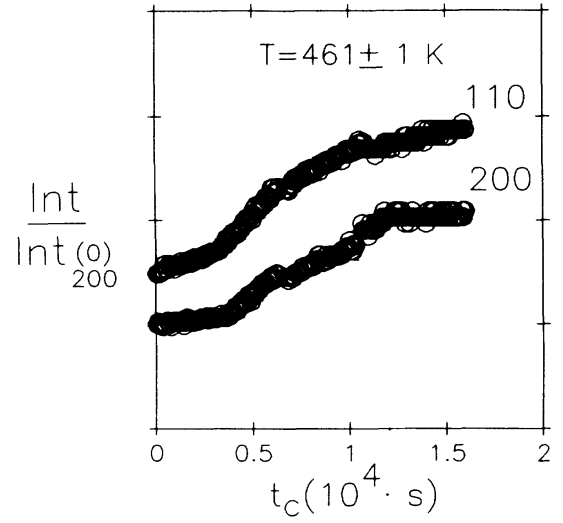

FIG. 6. Relative intensity (arbitrary units) of the 110 and 200 reflections as a function of crystallization time $\left(t_{c}\right)$ at $\mathrm{T}=461 \mathrm{~K}$ of the PEKK (60/40) system.

at $T_{c}=461 \pm 1 \mathrm{~K}$. The $\mathrm{x}$-ray scattered intensity is represented as a function of the reciprocal lattice vector $\mathbf{s}=(2 \sin \theta) / \lambda$. As crystallization time increases the $110\left(\mathrm{~s}=2.09 \mathrm{~nm}^{-1}\right)$ and $200\left(\mathrm{~s}=2.58 \mathrm{~nm}^{-1}\right)$ reflections, characteristic of a two-chain orthorhombic unit cell, appear. ${ }^{21}$

The relative intensity of the 110 and 200 reflections is plotted as a function of crystallization time in Fig. 6. After an induction time of about $3 \times 10^{3} \mathrm{~s}$ the relative intensities increase with $t_{c}$ to leveling off finally for times longer than $12 \times 10^{3} \mathrm{~s}$. The weight fraction crystallinity was estimated from the ratio between the crystalline peaks to the total area of coherent scattering af-

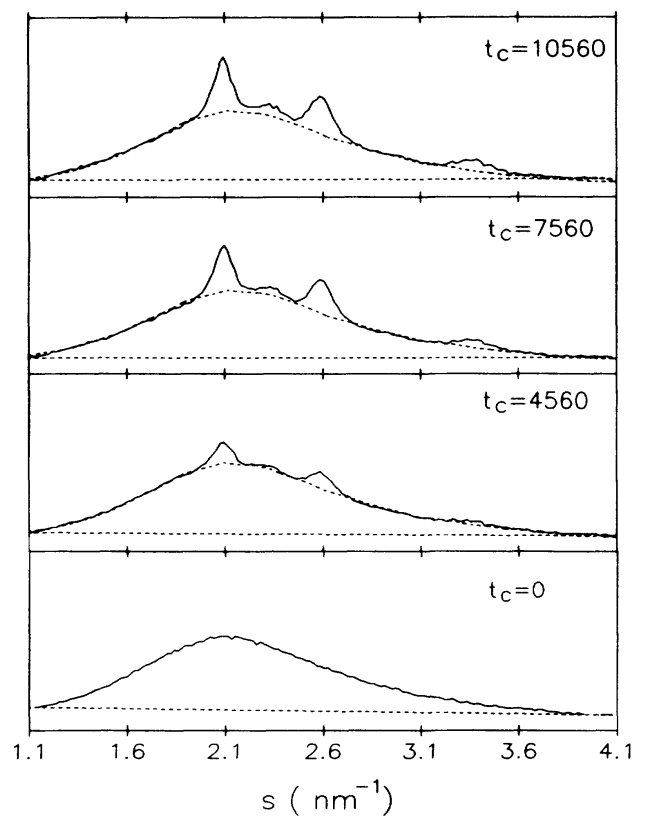

FIG. 7. WAXS patterns, as a function of $s=(2 \sin \theta) / \lambda$, taken at selected crystallization times at $T=461 \mathrm{~K}$ of the PEKK (60/40) system. The contribution of the amorphous phase at every crystallization time is represented by the dashed lines. ter subtraction of the incoherent background. ${ }^{24,25}$ Figure 7 illustrates the WAXS patterns, as a function of $\mathbf{s}=(2 \sin \theta) / \lambda$, taken at selected crystallization times. The contribution of the amorphous phase at every crystallization time (dashed lines in Fig. 7) was calculated by multiplying the initial scattering profile $\left(t_{c}=0\right)$ by a reduction factor and, then, shifting the obtained pattern along the $s$-axis till a good fit is achieved. Crystallinity values of $0 \%, 3 \%, 10 \%$, and $20 \%$ with an error of $\pm 2 \%$ are obtained for the diffraction patterns shown in Fig. 7.

\section{Phenomenological analysis of the experimental results}

Figure 8 shows the change of the Cole-Cole plots $\left(\epsilon^{\prime \prime}\right.$ vs $\epsilon^{\prime}$ ) corresponding to the $\alpha$ relaxation during the early stages of crystallization $\left(t_{c}<6.06 \times 10^{3} \mathrm{~s}\right)$. The solid lines correspond to the calculated fittings according to the HN equations [Eqs. (4)-(7)]. A subroutine based on the Newton method ${ }^{26}$ was modified to fulfil our requirements and used in the fitting. The parameters calculated in Fig. 8 are shown in Table I. To estimate the accuracy of the fitting parameters, their values have been varied. We found that the maximum possible variation possible without provoking a significant deviation between the measured and calculated curves was less than $\pm 9 \%$ for $b$, $c$ and $\Delta \epsilon$, and $\pm 10 \%$ for $\tau_{0}$.

From the present results the following features can be

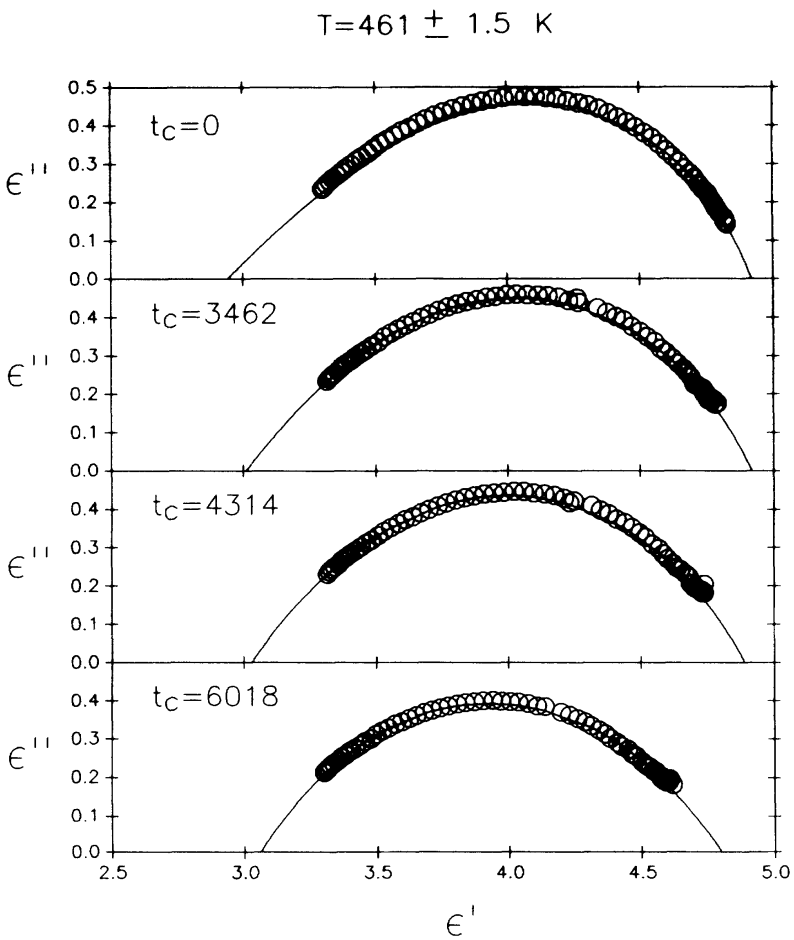

FIG. 8. Cole-Cole diagrams of the $\alpha$ relaxation for selected crystallization times $\left(t_{c}\right)$ at $T_{c}=461 \pm 1.5 \mathrm{~K}$. Solid curves denote best fits calculated according to Eqs. (4)-(7). Parameters of the fits are shown in Table $I$. 
TABLE I. $t_{c}, \Delta \epsilon, b, c$, and $\tau_{0}$ obtained from the fitting of Eqs. (4)-(7) for the $\alpha$ relaxation data shown in Fig. 8.

\begin{tabular}{c|c|c|c|c}
\hline \hline$t_{c}\left(10^{3} \mathrm{~s}\right)$ & $\Delta \epsilon$ & $b$ & $c$ & $\tau_{0}(\mathrm{~s})$ \\
\hline 0 & 1.98 & 0.69 & 0.55 & $1 \times 10^{-5}$ \\
\hline 3.462 & 1.91 & 0.62 & 0.74 & $6.2 \times 10^{-6}$ \\
\hline 4.314 & 1.86 & 0.59 & 0.83 & $5 \times 10^{-6}$ \\
\hline 6.018 & 1.7 & 0.54 & 1 & $3.8 \times 10^{-6}$ \\
\hline \hline
\end{tabular}

identified. First, a reduction of the high frequency skewness of the relaxation process as denoted by the increase of the $c$ parameter towards the highest possible value $(c=1)$. Second, a broadening increase of the relaxation curves as revealed by reduction of the $b$ parameter, and third, a decrease of the relaxation strength $\Delta \epsilon$. To analyze the complete real time evolution of the $\alpha$ relaxation process upon crystallization, the characteristic parameters of the HN formulation are shown in Fig. 9 as a function of crystallization time. It is seen that $\Delta \epsilon$ conspicuously decreases at earlier stages of crystallization and it levels off at longer crystallization times. A similar behavior is shown by the symmetric broadening parameter $b$. On the other hand, the nonsymmetric broadening parameter $c$, increases up to the maximum value of 1 indicating that the relaxation curves become symmetric as crystallization proceeds. The central relaxation time $\tau_{0}$, first, decreases for short times showing a minimum value at about $9 \times 10^{3} \mathrm{~s}$, then it bends upwards, and, finally, it levels off as crystallization time increases.

To obtain the change of the dipole moment time correlation function corresponding to the $\alpha$ process with crystallization time, Eq. (9) has been numerically solved.

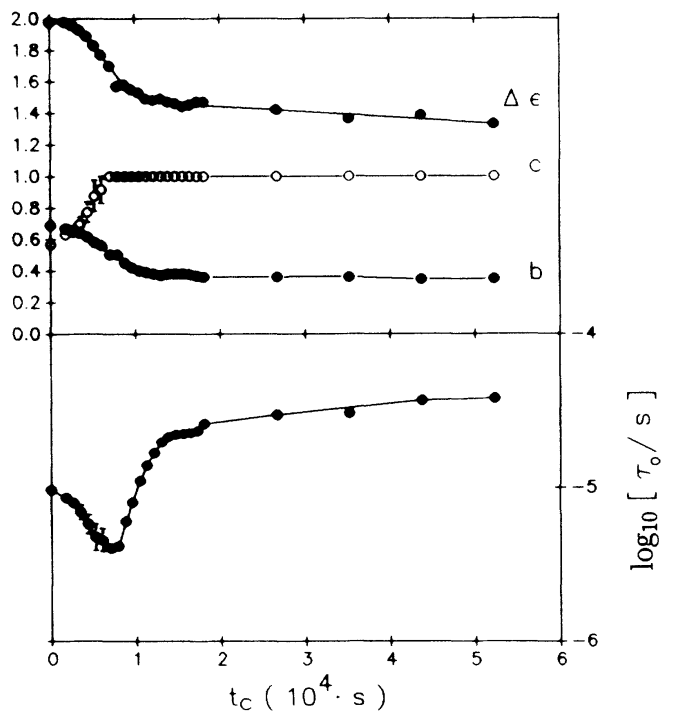

FIG. 9. Real-time evolution of $\Delta \epsilon, b$, and $c$ (upper) and central relaxation time $\tau_{0}$ during the isothermic crystallization process. Solid lines are guides to the eyes.

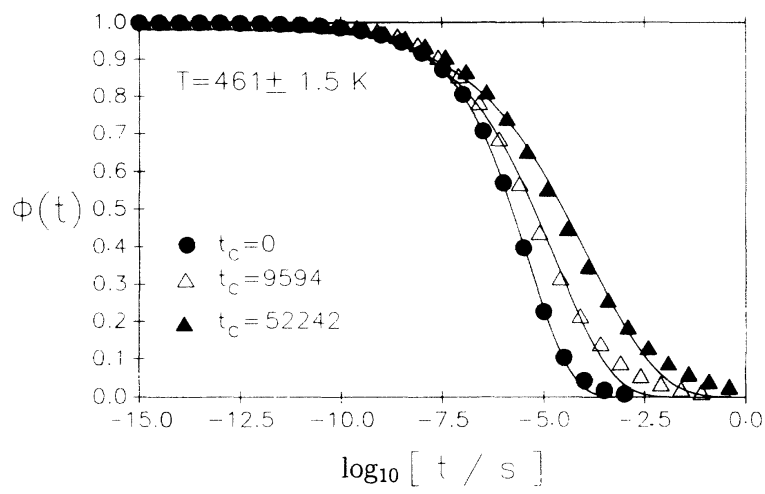

FIG. 10. Normalized $\phi(t)$ functions corresponding to different crystallization times as a function of $\log _{10}[t]$. The solid lines represent the best fits of the calculated data by the Kohlrausch-Williams-Watt function [Eq. (3)].

The $\epsilon^{\prime \prime}(\omega)$ values were generated from Eqs. (5)-(7) using the HN parameters corresponding to each crystallization time (Fig. 9). A subroutine based on the Hagenah procedure ${ }^{9}$ was developed to perform the inverse Laplace transformation. As an example, the normalized $\phi(t)$ functions corresponding to different crystallization times are represented in Fig. 10 as a function of $\log _{10}[t]$. The solid lines represent the best fit of the obtained values by the Kohlrausch-Williams-Watt function [Eq. (3)]. The corresponding fitting parameters are shown in Table II. The complete series of both stretching parameter $\beta$ and characteristic relaxation time $\tau_{\mathrm{KWW}}$ values as a function of the crystallization time is represented in Fig. 11. Additionally, the real-time variation of the mean relaxation time $\langle\tau\rangle$ calculated from Eq. (11) is also shown in Fig. 11. The variation of $\beta$ with $t_{c}$ at the initial stage remains almost unchanged, but it subsequently decreases and finally it levels off for longer crystallization times. In contrast, the characteristic relaxation time $\tau_{\mathrm{KWW}}$ remains practically unchanged for the initial times, then subsequently increases towards higher values, and finally levels off for higher crystallization times.

\section{DISCUSSION}

\section{A. Correlation of $\Delta \epsilon$ and crystallinity}

It is known that PEKK crystallizes through formation of spherulites. ${ }^{21}$ The sigmoidal-shape variation of the magnitudes presented in Figs. 6, 9, and 11 seems to

TABLE II. Fitting parameters corresponding to the KWW stretched exponentials of different crystallization times presented in Fig. 10.

\begin{tabular}{c||c|c}
\hline \hline$t_{c}\left(10^{4} \mathrm{~s}\right)$ & $\beta$ & $\tau_{\mathrm{KWw}}(\mathrm{s})$ \\
\hline 0 & 0.41 & $4 \times 10^{-6}$ \\
\hline 0.9594 & 0.307 & $1.9 \times 10^{-5}$ \\
\hline 5.2242 & 0.25 & $1.3 \times 10^{-4}$ \\
\hline \hline
\end{tabular}




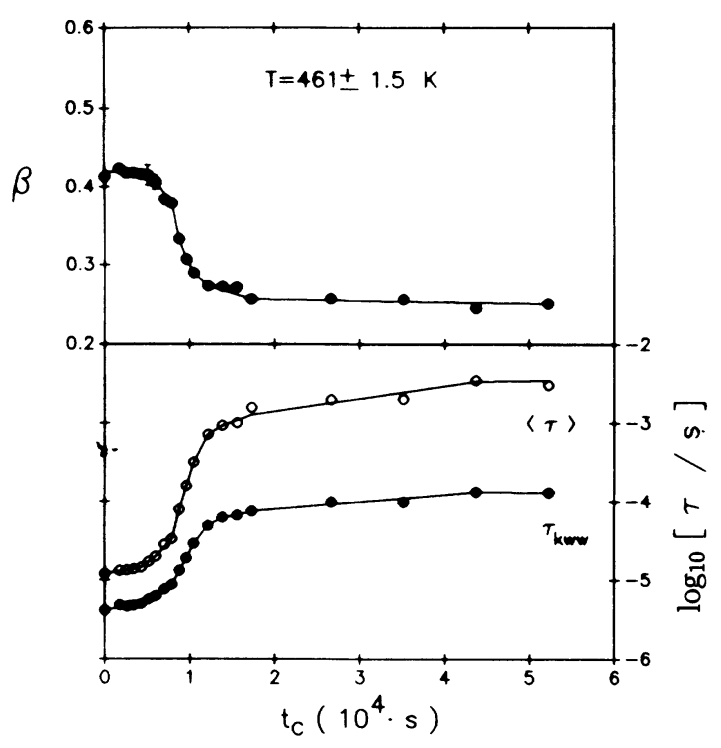

FIG. 11. Real-time evolution of the stretching parameter $\beta$ (upper), the characteristic relaxation time $\tau_{\mathrm{KWW}}$ and the mean relaxation time $\langle\tau\rangle$ as a function of the crystallization time $t_{c}$. The solid lines are a guide to the eye.

follow the characteristic features of an isothermal crystallization process as pointed out in the introduction. A particularly interesting result is that the variation of the dielectric strength (Fig. 9) decreases about $30 \%$ its initial value and levels off at times longer than about $12 \times 10^{3}$ s. Comparison of Figs. 6 and 9 shows a corresponding behavior between the variation of the relative intensity of the 110 and 200 reflections and that of $\Delta \epsilon$ values with crystallization time. Both experiments allow one to estimate a value for the half time of crystallization of about $6 \times 10^{3} \mathrm{~s}$.

Since $\Delta \epsilon$ is proportional to the density of dipoles involved in the $\alpha$ relaxation, ${ }^{27}$ the decrease of $\Delta \epsilon$ with crystallization time can be associated with the progressive reduction of the amorphous phase as segments of the polymeric chains crystallize. Figure 12 shows the corre-

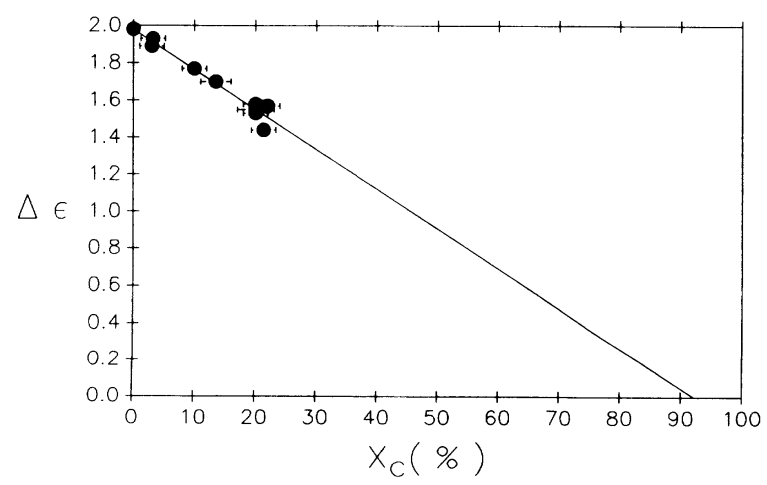

FIG. 12. Variation of $\Delta \epsilon$ with crystallinity as derived from WAXS experiments. The straight line represents the best linear dependence to the experimental data. lation found between $\Delta \epsilon$ and the x-ray crystallinity. The straight line drawn represents the best linear fit to the experimental data. As it is shown, the straight line extrapolates to $\Delta \epsilon=0$ at a crystallinity value different from $100 \%$. The enhanced reduction of $\Delta \epsilon$ with increasing crystallinity has been previously reported. ${ }^{14}$ This finding suggests that the restriction in motion of polymeric chains in the amorphous regions due to adjacent crystalline regions is higher than the expected one on the only basis of the amorphous content. This result indicates that for x-ray crystallinities of nearly $90 \%$ the molecular mobility, as deduced from $\Delta \epsilon$, should be negligible. From this result one may infer in agreement with other authors, ${ }^{28-30}$ the existence of a tightly bound amorphous phase which appears as a consequence of constrains of these rigid amorphous chains provoked by the influence of the crystal lamellae.

\section{B. Distribution of relaxation times during the crystallization process}

The observed evolution of the $\alpha$ relaxation process in real time, as analyzed by the $\mathrm{HN}$ approach, indicates the modification of the time distribution function during the isothermic crystallization process. For the short crystallization times the distribution of relaxation times for the $\alpha$ process is narrow and strongly asymmetric, as implied from the relatively low $b$ and $c$ initial values respectively (Fig. 9). As crystallinity develops the distribution of relaxation times becomes broader and tends to be more symmetric as revealed by the concurrent decrease of $b$ and increase of the $c$ values respectively. As the system further crystallizes the $\alpha$ relaxation process is increasingly restricted to the interlamellar amorphous regions. In addition, segments of the polymeric chains included in the crystals can hinder the overall mobility of bonded segments which remain in the amorphous phase by restricting the available possible conformations. ${ }^{15}$ Thus, the presence of crystals is more likely to affect large, rather than small scale motions. The experimentally observed variation of the $b$ and $c$ parameters in this scenario is in good accordance with the predictions of the SS model. The observed decrease of $b$ could be interpreted, according to this model, as due to the increasing hindrance of the large scale motions as crystallization proceeds. On the other hand, the value of $c \times b$ does not change substantially with crystallization time. This suggests, on the basis of the SS model, that the small scale motions are indeed less affected by the crystallization process.

\section{Influence of crystallinity \\ on the dipole moment time correlation function}

As shown in Fig. 10 the dipole moment time correlation function is also affected by the crystallization process. The normalized $\phi(t)$ function can be well described in terms of the $\mathrm{KWW}$ function although systematic deviations are observed at higher times as crystallinity increases. It has been noted that there are coupling $b$ and $c$ parameters from the HN formulation which may 
be univocally related to a single $\beta$ stretching parameter from the KWW function. ${ }^{2,10,11}$ However, computer calculations have shown ${ }^{11}$ that for symmetrically broadened relaxation curves $(b<0.4, c=1)$ the equivalence between the $\mathrm{HN}$ and the KWW functions becomes less accurate. Since the $\alpha$ relaxation in semicrystalline polymers is usually broad and symmetric, the question arises as whether the two HN parameters which describe the $\alpha$ relaxation in semicrystalline polymers can also be described with an unique $\mathrm{KWW}$ function in the time domain as seems to occur in amorphous polymers. ${ }^{31}$

We find that the calculated $\beta$ parameter decreases as crystallization proceeds (Fig. 11). According to the coupling model, a decrease of the $\beta=1-n$ parameter is expected provided that a strengthening of the intermolecular coupling between the relaxing species occurs. It has been shown ${ }^{32}$ that intramolecular coupling provoking restrictions of conformational transitions may also affect the intermolecular coupling by increasing the intermolecular cooperativity. In the case of semicrystalline polymers the connection of chain segments in the amorphous phase to the crystals increase the cooperativity of these segments by reducing the number of conformations. ${ }^{15}$ Consequently, we may interpret the observed decrease of $\beta$, within this context, as a consequence of the increase of interchain coupling between the relaxing units caused by the additional intrachain constrains introduced by the crystallinity. A similar influence of crystallinity on the molecular mobility in poly(ethylene oxide) as revealed by mechanic dynamical measurements has been recently reported. ${ }^{33}$ It is expected that the increase of interchain coupling suffered by a polymeric segment upon crystallization will depend on its chemical structure. For example, from the analysis of the temperature dependence of the relaxation time it has been shown ${ }^{34}$ that a flexible polymer like poly(propylene) exhibits a similar degree of intermolecular coupling both as amorphous and as semicrystalline material. On the other hand, a stiffer polymer like poly(ethylene terephthalate) exhibits a slightly different behavior when comparing the amorphous and the semicrystalline samples even though this observed difference was claimed to be small. ${ }^{32,34}$ Exten- sion of the present studies to other polymeric systems will help to understand the physical mechanisms affecting the dynamics of the $\alpha$ relaxation in semicrystalline polymers

\section{CONCLUSIONS}

Dielectric relaxation measurements can be used to characterize the changes occurring in glassy polymers during isothermal crystallization in real time. The variation of the dielectric parameters, particularly the dielectric strength, follows a sigmoidal-shaped pattern that is characteristic of an isothermal crystallization process of a polymeric material. The changes observed in the dielectric relaxation process during crystallization can be phenomenologically described in terms of the HavriliakNegami approximation. The $\alpha$ relaxation broadens and becomes symmetric as crystallization proceeds. This evolution has been interpreted, according to the SchönhalsSchlosser-model, by the restriction of the long scale motions of the polymeric chains as the material is filled in with crystals. The dipole moment time correlation functions can be well described by the KWW function. However, as predicted by computer calculations, the equivalence between $\mathrm{HN}$ and $\mathrm{KWW}$ functions becomes less accurate as the relaxation becomes broader and symmetric. The calculated mean relaxation time for the $\alpha$ relaxation increases with crystallization time suggesting a progressive reduction of overall mobility of the polymeric chains as the system becomes more crystalline. Concurrently, an increase of the stretching of KWW function is detected which has been qualitatively interpreted by the increase of the interchain coupling between the relaxing species in the amorphous phase.

\section{ACKNOWLEDGMENTS}

The authors are indebted to the CICYT (Grant No. MAT 94-0740E), Spain, for generous support of this investigation. J.M. thanks the Tempus Program (JEP 0644) for the support of his investigations.
* Permanent address: Institute of Physics, Polytechnical University of Szczecin, 70-310 Szczecin, Poland.

${ }^{1}$ S. Havriliak and S. Negami, Polymer 8, 161 (1967).

${ }^{2}$ J. Colmenero, A. Alegria, J.M. Alberdi, F. Alvarez, and B. Frick, Phys. Rev. B 44, 7321 (1991).

${ }^{3}$ J. Colmenero, A. Alegria, A. Arbe, and B. Frik, Phys. Rev. Lett. 69, 478 (1992).

${ }^{4}$ A. Schönhals and E. Schlosser, Colloid Polym. Sci. 267, 125 (1989).

${ }^{5}$ A. Schönhals, F. Kremer, and E. Schlosser, Phys. Rev. Lett. 67, 999 (1991).

${ }^{6}$ G. Williams and D.C. Watts, Trans. Faraday Soc. 66, 80 (1970).

${ }^{7}$ K.L. Ngai and C.T. White, Phys. Rev. B 20, 2475 (1979).

${ }^{8}$ K.L. Ngai, A.K. Rajagopal, and S. Teiler, J. Chem. Phys. 88, 5086 (1988).
${ }^{9}$ D. Boese, B. Momper, G. Meier, F. Kremer, J.U. Hagenah, and E.W. Fischer, Macromolecules 22, 4416 (1989).

${ }^{10}$ F. Alvarez, A. Alegria, and J. Colmenero, Phys. Rev. B 44, 7306 (1991).

${ }^{11}$ F. Alvarez, A. Alegria, and J. Colmenero, Phys. Rev. B 47, 125 (1993).

${ }^{12}$ L. Mandelkern, Crystallization of Polymers (McGraw-Hill, New York, 1964).

${ }^{13}$ J.M. Schultz, Polymer Materials Science (Prentice-Hall, New York, 1974), p. 380.

${ }^{14}$ G. Williams, Adv. Polym. Sci. 33, 59 (1979).

${ }^{15}$ J.C. Coburn and R.H. Boyd, Macromolecules 19, 2238 (1986).

${ }^{16}$ E. Schlosser and A. Schönhals, Colloid Polym. Sci. 267, 963 (1989).

${ }^{17}$ K.U. Kirst, F. Kremer, and V.M. Litvinov, Macromolecules 
26, 975 (1993).

${ }^{18}$ F. Kremer, D. Boese, G. Meier, and E.W. Fischer, Prog. Colloid Polym. Sci. 80, 129 (1989).

${ }^{19}$ M. Cook, D.C. Watts, and G. Williams, Trans. Faraday Soc. 66, 2503 (1970).

${ }^{20} \mathrm{G}$. Williams, in Structure and Properties of Polymers, Materials Science and Technology Vol. 12, edited by R.W. Cahn, P. Haasen, and E.J. Kramer (VCH, Weinheim, 1993), p. 471.

${ }^{21}$ K.H. Gardner, B. S. Hsiao, R.R. Matheson, Jr., and B.A. Wood, Polymer 33, 2483 (1992).

${ }^{22}$ C. Boulin, R. Kempf, M.H.J. Koch, and S.M. Mclaughlin, Nucl. Instrum. Methods Phys. Res. A 249, 399 (1986).

${ }^{23}$ B.B. Sauer, P. Avakian, H.W. Starkweather, Jr., and B. Hsiao, Macromolecules 23, 5119 (1990).

${ }^{24}$ D.J. Blundell and B.N. Osborn, Polymer 24, 953 (1983).

${ }^{25}$ F.J. Balta-Calleja and C.G. Vonk, X-ray Scattering of Synthetic Polymers (Elsevier, Amsterdam, 1989).
${ }^{26}$ K. Ebert, H. Ederer, and T.L. Isenhour, Computer Applications in Chemistry (VCH, Weinheim, 1989).

${ }^{27}$ P. Hedvig, Dielectric Spectroscopy of Polymers (Adam Hilger Ltd., Bristol, 1977).

${ }^{28}$ S.Z.D. Cheng, M.Y. Cao, and B. Wunderlich, Macromolecules 19, 1868 (1986).

${ }^{29}$ P.P. Huo and P. Cebe, Polymer 34, 696 (1993).

${ }^{30}$ P.P. Huo, J. B. Friler, and P. Cebe, Polymer 34, 4387 (1993).

${ }^{31}$ R.H. Boyd, R.W. Devereaux, and R.B. Thayer, Polym. Preprints 31, 279 (1990).

${ }^{32}$ K.L. Ngai and C.M. Roland, Macromolecules 26, 6824 (1993).

${ }^{33}$ A. Bartolotta, G. Di marco, M. Lanza, and G. Carini, Phys. Rev. B 48, 10137 (1993).

${ }^{34}$ K.L. Ngai and C.M. Roland, Macromolecules (to be published). 